\title{
Linear analysis of Hamilton perturbation theory for coupled betatron motion
}

\author{
M. Takao* \\ SPring-8, 1-1-1 Kouto, Sayo, Hyogo 679-5198, Japan
}

(Received 3 March 2006; published 30 August 2006)

\begin{abstract}
The dynamics of coupled betatron motion in a circular accelerator has been studied by the Hamilton perturbation theory or the transfer matrix formalism separately. In order to incorporate the theories with each other, we develop the transfer matrix formulation on the Hamilton perturbation theory. With the help of the Hamilton perturbation theory, we can analytically solve the equation of the coupled betatron motion in the vicinity of a difference resonance. Using the solution, we can construct the transfer matrix for the coupled motion. The matrix is shown to be symplectic and then transformed to the block diagonal form which consists of two normal modes and the coupling matrix. Thus, we derive the analytical representation for the normal mode parametrization of the transfer matrix describing the coupled betatron motion. Using the formalism, we investigate the two-dimensional beam ellipse in an electron storage ring.
\end{abstract}

DOI: 10.1103/PhysRevSTAB.9.084002

PACS numbers: 29.27. $-\mathrm{a}, 41.85 .-\mathrm{p}$

\section{INTRODUCTION}

In circular accelerators the correct compensation of the betatron coupling is essential for the ring operation and for the performance. Hence, the betatron coupling has been intensively studied by various methods. The Hamilton perturbation theory [1-4] is one of the most practical methods to investigate the coupled betatron motion, which can solve the equations of motion analytically. Although the Hamilton perturbation theory provides a good physical insight, it leaves the lattice structure of the coupled motion obscure. On the other hand, in the transfer matrix formalism the transverse beam motion is parametrized by a 4-by4 matrix. Since the matrix satisfies the symplectic condition required by the Hamilton equation of motion, it is decomposed into block diagonal form consisting of the respective Twiss functions [5,6] and a 2-by-2 coupling matrix $[7,8]$. However, it is not straightforward for the matrix formalism to assign analytical expressions to the coupling parameters and the tune shifts for the coupling source distribution.

In this paper, for the purpose of amalgamating both the formalism with adopting the respective virtues, we complete the transfer matrix formulation on the Hamilton perturbation theory. In the latter theory, we have already known the solution of the equation of coupled betatron motion, from which we can construct the transfer matrix. Of course the matrix is symplectic, and then can be decomposed into the block diagonal form. Thus, we derive the analytical representation of the normal modes and the coupling matrix. There were attempts to combine the Hamiltonian and matrix treatment [9-12], where the relation between the Hamiltonian terms, i.e., the coupling driving terms, and the coupling matrix was established. In the present paper we derive the representation of the transfer matrix in terms of the solution of the equation of

*Electronic address: takao@spring8.or.jp motion and give clear expressions for the matrix parametrization.

Applying the formulation to an electron ring, we investigate the beam parameters of two-dimensional coupled motion. To reveal the effectiveness, we measure the twodimensional beam profile of the SPring- 8 storage ring by comparing the present formulation.

In Sec. II we briefly review the Hamilton perturbation theory [1-4] to define the notation. Then, in Sec. III, the transfer matrix formulation is completed based on the solution derived by the Hamilton perturbation theory. As an application of the present formulation, we derive the beam envelope under coupled motion in an electron storage ring in Sec. IV.

\section{HAMILTONIAN TREATMENT OF LINEAR COUPLING}

We consider a linear lattice with small skew quadrupole distortions distributed over the circumference of a circular accelerator. The form of the Hamiltonian $H$ describing the linearly coupled system can be written as

$$
H=\frac{1}{2}\left[p_{x}^{2}+p_{y}^{2}+G_{x}(s) x^{2}+G_{y}(s) y^{2}+2 K(s) x y\right],
$$

where $G_{x, y}$ are the forces exerted on the particles by the magnetic gradients, $K$ the strength of the skew quadrupole magnet, and $s$ the path length. Knowing the Hamiltonian for the betatron motion $H_{0}$

$$
H_{0}=\frac{1}{2}\left[p_{x}^{2}+p_{y}^{2}+G_{x}(s) x^{2}+G_{y}(s) y^{2}\right],
$$

the perturbed Hamiltonian for linear coupling $H_{1}$ is obtained by subtracting $H_{0}$ from $H$ :

$$
H_{1}\left(x, p_{x}, y, p_{y}\right)=K(s) x y .
$$

Suppose we have solved the unperturbed problem described by $H_{0}$ with the solution expressed in terms of the unperturbed lattice functions 


$$
z_{0}(s)=a_{z} w_{0}(s)+\text { c.c., } \quad p_{z, 0}(s)=a_{z} w_{0}^{\prime}(s)+\text { c.c., }
$$

where c.c. denotes complex conjugate of the preceding term and $w_{0}$ is a solution for unperturbed betatron motion:

$$
\begin{gathered}
w_{0}(s) \equiv \sqrt{\frac{\beta_{z}(s)}{2}} e^{i \phi_{z}(s)}, \\
\phi_{z}(s) \equiv \int_{0}^{s} \frac{d \tilde{s}}{\beta_{z}(\tilde{s})}
\end{gathered}
$$

with $w=u, v$ for $z=x, y$, respectively. Note that in the unperturbed system the complex amplitude $a_{z}$ 's are constants of motion.

Now we represent the solution for the full Hamiltonian in terms of the same lattice functions. Hence, we allow the constant $a_{z}$ 's become dependent on $s$ :

$$
\begin{aligned}
& z(s)=a_{z}(s) w_{0}(s)+\text { c.c. } \\
& p_{z}(s)=a_{z}(s) w_{0}^{\prime}(s)+\text { c.c. }
\end{aligned}
$$

Here it should be emphasized that, since the changes of the amplitudes are much slower than the betatron oscillation, we neglect the derivatives of $a_{z}$ 's compared to those of $w_{0}$ in $p_{z}$. We express the perturbing Hamiltonian (1) in terms of new variables $a_{z}$ 's:

$$
H_{1}=\left[h_{+}(s) a_{x} a_{y}+\text { c.c. }\right]+\left[h_{-}(s) a_{x} \bar{a}_{y}+\text { c.c. }\right],
$$

where

$$
h_{ \pm}(s)=\frac{1}{2} K(s) \sqrt{\beta_{x}(s) \beta_{y}(s)} e^{i\left[\phi_{x}(s) \pm \phi_{y}(s)\right]} .
$$

The source of the perturbing Hamiltonian is random error of optics functions so that in the most cases the coupling effect distorts the beam behavior very little. But if the errors resonate to the beam motion, the small distortion can act coherently on the beam and give the significant effect.

In the following, we investigate the dynamics near the resonance. In order to derive the resonant condition, we separate $h_{ \pm}$into a periodic and a nonperiodic factors,

$$
h_{ \pm}(s)=\tilde{h}_{ \pm}(s) e^{2 \pi i\left(\nu_{x} \pm \nu_{y}\right) s / L},
$$

where the periodic factors $\tilde{h}_{ \pm}$are given by

$$
\tilde{h}_{ \pm}(s)=\frac{K(s)}{2} \sqrt{\beta_{x}(s) \beta_{y}(s)} e^{i\left[\phi_{x}(s) \pm \phi_{y}(s)-\frac{2 \pi s}{L}\left(\nu_{x} \pm \nu_{y}\right)\right]}
$$

with the circumference $L$ and the betatron tunes $\nu_{z}=$ $\phi_{z}(L) /(2 \pi)$. The periodic factors $\tilde{h}_{ \pm}$can be represented by the Fourier coefficients,

$$
\begin{aligned}
& \tilde{h}_{ \pm}(s)=\sum_{q} \hat{h}_{ \pm}(q) e^{-2 \pi i q s / L}, \\
& \hat{h}_{ \pm}(q)=\frac{1}{L} \int_{0}^{L} d s \tilde{h}_{ \pm}(s) e^{2 \pi i q s / L},
\end{aligned}
$$

which are called driving terms. The perturbing Hamiltonian then has the form

$$
\begin{aligned}
H_{1}= & \sum_{q} \hat{h}_{+}(q) a_{x} a_{y} e^{2 \pi i\left(\nu_{x}+\nu_{y}-q\right) s / L}+\text { c.c. } \\
& +\sum_{q} \hat{h}_{-}(q) a_{x} \bar{a}_{y} e^{2 \pi i\left(\nu_{x}-\nu_{y}-q\right) s / L}+\text { c.c. }
\end{aligned}
$$

Now we find that the perturbing Hamiltonian oscillates with tunes $\nu_{x} \pm \nu_{y}-q$. Since $K$ is assumed to be small, the driving terms are a small perturbation only. Thus, they can induce only a slow change in the amplitudes $a_{z}$ 's, and the terms with quick oscillating frequency cannot have much influence on the amplitudes due to the fact that they average away before making a significant change. Only terms with small $\nu_{x} \pm \nu_{y}-q$ can act for long enough coherently on the variables and cause serious effects. Such terms are only present if the tunes satisfy a resonant condition,

$$
\nu_{x} \pm \nu_{y} \approx q(=\text { integer }) .
$$

As a consequence we only consider resonant cases.

In the following, we will only discuss the dynamics of the difference resonance. For the first order (in the perturbation function) resonances, coupling coefficients proportional to the driving terms can be written by definition

$$
C=\frac{L}{\pi} \hat{h}_{-}(q)
$$

with, in addition,

$$
\Delta=\nu_{x}-\nu_{y}-q,
$$

which has the meaning of a distance from the resonance.

The solution of the coupling motion is derived as follows. The perturbing Hamiltonian $H_{1}$ with neglecting the sum resonance can be written in terms of $C$ and $\Delta$ as

$$
H_{1}=\frac{\pi}{L}\left(C a_{x} \bar{a}_{y} e^{2 \pi i \Delta s / L}+\bar{C} \bar{a}_{x} a_{y} e^{-2 \pi i \Delta s / L}\right) .
$$

Solving the equation of motion for $a_{x, y}$ 's derived by the above perturbing Hamiltonian, one obtains

$$
\begin{gathered}
a_{x}(s)=A_{1} e^{-2 \pi i \nu_{1} s / L}+A_{2} e^{-2 \pi i \nu_{2} s / L}, \\
a_{y}(s)=\frac{C}{2}\left(\frac{A_{1}}{\nu_{2}} e^{2 \pi i \nu_{2} s / L}+\frac{A_{2}}{\nu_{1}} e^{2 \pi i \nu_{1} s / L}\right) .
\end{gathered}
$$

where $A_{1,2}$ 's are integration constants, and

$$
\nu_{1,2}=\frac{1}{2}\left(\Delta \pm \sqrt{\Delta^{2}+|C|^{2}}\right) .
$$

Note that $\nu_{1}>0$ and $\nu_{2}<0$ if $C \neq 0$.

\section{TRANSFER MATRIX}

Under the perturbation assumptions (4) and (5) of slow varying amplitudes, we have solved the equation of motion 
and obtained the solution of the amplitude function (10) and (11). Inserting the solution into Eqs. (4) and (5), we have the relation between the coordinates and the integral constants

$$
\mathbb{X}(s)=\boldsymbol{W}(s) \mathbb{A},
$$

where

$$
\mathbb{X}=\left(\begin{array}{c}
x \\
p_{x} \\
y \\
p_{y}
\end{array}\right), \quad \mathbb{A}=\left(\begin{array}{c}
A_{1} \\
A_{2} \\
\bar{A}_{1} \\
\bar{A}_{2}
\end{array}\right),
$$

$$
\begin{aligned}
M_{x y}= & \frac{1}{2\left(\nu_{1}-\nu_{2}\right)}\left[N _ { x y } ^ { ( s ) } \left\{-\sin 2 \pi\left(\nu_{x}-\nu_{1}\right)\right.\right. \\
& \left.+\sin 2 \pi\left(\nu_{x}-\nu_{2}\right)\right\}+N_{x y}^{(c)}\left\{\cos 2 \pi\left(\nu_{x}-\nu_{1}\right)\right. \\
& \left.\left.-\cos 2 \pi\left(\nu_{x}-\nu_{2}\right)\right\}\right]
\end{aligned}
$$

$$
\begin{aligned}
M_{y x}= & \frac{1}{2\left(\nu_{1}-\nu_{2}\right)}\left[N_{y x}^{(s)}\left\{\sin 2 \pi\left(\nu_{y}+\nu_{1}\right)-\sin 2 \pi\left(\nu_{y}+\nu_{2}\right)\right\}\right. \\
& \left.+N_{y x}^{(c)}\left\{\cos 2 \pi\left(\nu_{y}+\nu_{1}\right)-\cos 2 \pi\left(\nu_{y}+\nu_{2}\right)\right\}\right],
\end{aligned}
$$

and

$$
\begin{aligned}
& M_{x 1,2}= I \cos 2 \pi\left(\nu_{x}-\nu_{1,2}\right)+J_{x} \sin 2 \pi\left(\nu_{x}-\nu_{1,2}\right), \\
& M_{y 1,2}= I \cos 2 \pi\left(\nu_{y}+\nu_{1,2}\right)+J_{y} \sin 2 \pi\left(\nu_{y}+\nu_{1,2}\right), \\
& N_{x y}^{(s)}=\left(\begin{array}{cc}
\sqrt{\frac{\beta_{x}}{\beta_{y}}}\left(c_{r} \alpha_{y}+c_{i}\right) & \sqrt{\beta_{x} \beta_{y}} c_{r} \\
-\frac{c_{r}\left(1+\alpha_{x} \alpha_{y}\right)+c_{i}\left(\alpha_{x}-\alpha_{y}\right)}{\sqrt{\beta_{x} \beta_{y}}} & -\sqrt{\frac{\beta_{y}}{\beta_{x}}}\left(c_{r} \alpha_{x}-c_{i}\right)
\end{array}\right), \\
& N_{x y}^{(c)}=\left(\begin{array}{cc}
-\sqrt{\frac{\beta_{x}}{\beta_{y}}}\left(c_{r}-c_{i} \alpha_{y}\right) & \sqrt{\beta_{x} \beta_{y}} c_{i} \\
\frac{c_{r}\left(\alpha_{x}-\alpha_{y}\right)-c_{i}\left(1+\alpha_{x} \alpha_{y}\right)}{\sqrt{\beta_{x} \beta_{y}}} & -\sqrt{\frac{\beta_{y}}{\beta_{x}}}\left(c_{r}+c_{i} \alpha_{x}\right)
\end{array}\right), \\
& N_{y x}^{(s)}=\left(\begin{array}{cc}
\sqrt{\frac{\beta_{y}}{\beta_{x}}}\left(c_{r} \alpha_{x}-c_{i}\right) & \sqrt{\beta_{x} \beta_{y}} c_{r} \\
-\frac{c_{r}\left(1+\alpha_{x} \alpha_{y}\right)+c_{i}\left(\alpha_{x}-\alpha_{y}\right)}{\sqrt{\beta_{x} \beta_{y}}} & -\sqrt{\frac{\beta_{x}}{\beta_{y}}}\left(c_{r} \alpha_{y}+c_{i}\right)
\end{array}\right), \\
& N_{y x}^{(c)}=\left(\begin{array}{cc}
\sqrt{\frac{\beta_{y}}{\beta_{x}}}\left(c_{r}+c_{i} \alpha_{x}\right) & \sqrt{\beta_{x} \beta_{y}} c_{i} \\
\frac{c_{r}\left(\alpha_{x}-\alpha_{y}\right)-c_{i}\left(1+\alpha_{x} \alpha_{y}\right)}{\sqrt{\beta_{x} \beta_{y}}} & \sqrt{\frac{\beta_{x}}{\beta_{y}}}\left(c_{r}-c_{i} \alpha_{y}\right)
\end{array}\right),
\end{aligned}
$$

$$
\begin{aligned}
W_{x} & =\left(\begin{array}{ll}
u_{0}(s) e^{-\left[\left(2 \pi i \nu_{1}\right) / L\right] s} & u_{0}(s) e^{-\left[\left(2 \pi i \nu_{2}\right) / L\right] s} \\
u_{0}^{\prime}(s) e^{-\left[\left(2 \pi i \nu_{1}\right) / L\right] s} & u_{0}^{\prime}(s) e^{-\left[\left(2 \pi i \nu_{2}\right) / L\right] s}
\end{array}\right), \\
W_{y} & =\left(\begin{array}{cc}
\frac{C}{2 \nu_{2}} \boldsymbol{v}_{0}(s) e^{\left[\left(2 \pi i \nu_{2}\right) / L\right] s} & \frac{C}{2 \nu_{1}} \boldsymbol{v}_{0}(s) e^{\left[\left(2 \pi i \nu_{1}\right) / L\right] s} \\
\frac{C}{2 \nu_{2}} \boldsymbol{v}_{0}^{\prime}(s) e^{\left[\left(2 \pi i \nu_{2}\right) / L\right] s} & \frac{C}{2 \nu_{1}} \boldsymbol{v}_{0}^{\prime}(s) e^{\left[\left(2 \pi i \nu_{1}\right) / L\right] s}
\end{array}\right) .
\end{aligned}
$$

In the above equations, $u_{0}(s)$ and $v_{0}(s)$ are the solutions for the horizontal and vertical unperturbed betatron motions (2), respectively. From now on, we denote the 4-by-4 (2by-2) matrix by the bold (light) capital letter.

Initially, i.e., at $s=0$, the relation (13) is simply

$$
\mathbb{X}(0)=\boldsymbol{W}(0) \mathbb{A} \text {. }
$$

For reasons of simplicity, it has been assumed in what follows that $\varphi_{z}(0)=0$. The inversion of the matrix $\boldsymbol{W}(0)$ gives the complex amplitudes in terms of the initial coordinates. Hence, we can get the transfer matrix $\boldsymbol{M}$ for one revolution by putting $s=L$ :

$$
\mathbb{X}(L)=\boldsymbol{M} \backslash(0) \equiv \boldsymbol{W}(L) \boldsymbol{W}^{-1}(0) \mathbb{X}(0),
$$

or

$$
\boldsymbol{M}=\boldsymbol{W}(L) \boldsymbol{W}^{-1}(0) .
$$

Thus, we represent the transfer matrix $\boldsymbol{M}$ in terms of the unperturbed lattice functions as well as $\boldsymbol{W}$. If we decompose the transfer matrix $\boldsymbol{M}$ into 2-by-2 matrices

$$
\boldsymbol{M}=\left(\begin{array}{ll}
M_{x x} & M_{x y} \\
M_{y x} & M_{y y}
\end{array}\right)
$$

the element matrices are given by

$$
\begin{aligned}
& M_{x x}=\frac{1}{\nu_{1}-\nu_{2}}\left(-\nu_{2} M_{x 1}+\nu_{1} M_{x 2}\right), \\
& M_{y y}=\frac{1}{\nu_{1}-\nu_{2}}\left(-\nu_{2} M_{y 1}+\nu_{1} M_{y 2}\right),
\end{aligned}
$$

where $I$ is the 2-by-2 unit matrix, and $J_{z}$ the Twiss matrices

$$
J_{z}=\left(\begin{array}{cc}
\alpha_{z} & \beta_{z} \\
-\gamma_{z} & -\alpha_{z}
\end{array}\right)
$$

$c_{r}$ and $c_{i}$, respectively, the real and imaginary parts of $C$. As found from the fact that the determinants of the matrices $M_{x 1,2}$ and $M_{y 1,2}$ are equal to 1 ,

$$
\operatorname{Det}\left(M_{x 1,2}\right)=\operatorname{Det}\left(M_{y 1,2}\right)=1 \text {, }
$$

they are identical to a transfer matrix in one dimension except for the slight variation in the tunes. It is an interesting fact that the matrices $M_{x x}$ and $M_{y y}$ are averages of the one-dimensional transfer matrices. The matrices $N_{x y}^{(s, c)}$ and $N_{y x}^{(s, c)}$ also have the constant determinants equal to the strength of the coupling:

$$
\operatorname{Det}\left(N_{x y}^{(s, c)}\right)=\operatorname{Det}\left(N_{y x}^{(s, c)}\right)=|C|^{2} .
$$

It is well known that the Hamilton theory requires that the transfer matrix must satisfy the symplectic condition $[7,8]$

$$
\boldsymbol{M}^{t} \boldsymbol{S} \boldsymbol{M}=\boldsymbol{S}
$$


where the superscript $t$ denotes the transposition of the matrix, and $S$ is the unit antisymmetric matrix

$$
S=\left(\begin{array}{cc}
S & O \\
O & S
\end{array}\right)
$$

with

$$
S=\left(\begin{array}{cc}
0 & 1 \\
-1 & 0
\end{array}\right), \quad O=\left(\begin{array}{cc}
0 & 0 \\
0 & 0
\end{array}\right) .
$$

After lengthy but straightforward calculation, we can show that the transfer matrix (14) satisfies the symplectic condition (20).

Following Edwards and Teng [7,8], we introduce the symplectic rotation matrix $\boldsymbol{T}$,

$$
\boldsymbol{T}=\left(\begin{array}{cc}
\tau I & -T^{*} \\
T & \tau I
\end{array}\right)
$$

with

$$
\tau^{2}+\operatorname{Det}(T)=1,
$$

which block diagonalizes the transfer matrix $\boldsymbol{M}$,

$$
\boldsymbol{T} \boldsymbol{M} \boldsymbol{T}^{-1}=\left(\begin{array}{ll}
U & O \\
O & V
\end{array}\right)
$$

In Eq. (22) the superscript $*$ denotes the adjoint operation defined by $T^{*}=-S T^{t} S$. The matrices $U$ and $V$ are unimodular ones corresponding to respective the eigenmode in two-dimensional oscillation. Equation (24) means that the transfer matrix is parametrized by the two unimodular matrices $U, V$ and the coupling matrix $T$, which is called Teng parametrization. This equation also implies that the coupled motion is completely decoupled in the coordinate transformed by the symplectic rotation matrix $\boldsymbol{T}$. Hence, the coordinate is called the normal one.

In order to get the normal coordinate for the given transfer matrix, we must solve the symplectic rotation matrix and the two eigenmode matrices in terms of the transfer matrix $\boldsymbol{M}$. After doing so, we obtain

$$
\tau^{2}=\frac{\nu_{1}}{\nu_{1}-\nu_{2}},-\frac{\nu_{2}}{\nu_{1}-\nu_{2}}
$$

In the case of $\Delta \geq 0$, we choose the former solution in order to get $\tau \rightarrow 1$ at far from resonance $\Delta \gg|C|$. Hence, we obtain the symplectic rotation matrix for $\Delta \geq 0$ :

$$
T=-\frac{\tau}{2 \nu_{1}} N_{y x}^{(c)} .
$$

After all, the 4-by-4 symplectic rotation matrix is given by

$$
\boldsymbol{T}=\tau\left(\begin{array}{cc}
I & -\frac{1}{2 \nu_{1}} N_{x y}^{(c)} \\
-\frac{1}{2 \nu_{1}} N_{y x}^{(c)} & I
\end{array}\right) .
$$

By simply using the above representation for $\boldsymbol{T}$ in Eq. (24), we can show that the transfer matrix $\boldsymbol{M}$ is diagonalized as follows:

$$
\boldsymbol{T} \boldsymbol{M} \boldsymbol{T}^{-1}=\left(\begin{array}{cc}
M_{x 2} & 0 \\
0 & M_{y 2}
\end{array}\right) .
$$

In the case of $\Delta<0$, we choose $\tau=\sqrt{-\nu_{2} /\left(\nu_{1}-\nu_{2}\right)}$ to get $\tau \rightarrow 1$ at far from resonance. In this case we have the symplectic rotation matrix

$$
\boldsymbol{T}=\tau\left(\begin{array}{cc}
I & -\frac{1}{2 \nu_{2}} N_{x y}^{(c)} \\
-\frac{1}{2 \nu_{2}} N_{y x}^{(c)} & I
\end{array}\right)
$$

and the normal form of the transfer matrix

$$
\boldsymbol{T} \boldsymbol{M} \boldsymbol{T}^{-1}=\left(\begin{array}{cc}
M_{x 1} & 0 \\
0 & M_{y 1}
\end{array}\right) .
$$

In this way we complete the matrix formulation for the Hamilton perturbation theory for coupled betatron motion. Then we give the analytic representation for the coupling matrix of Teng parametrization.

The eigentunes in the normal coordinate for $\Delta \geq 0$ are thus

$$
\nu_{u, v}=\nu_{x, y} \mp \nu_{2},
$$

and those for $\Delta<0$

$$
\nu_{u, v}=\nu_{x, y} \mp \nu_{1},
$$

which are the measured ones in an actual experiment. Note that hereafter we denote the eigenmodes by $(u, v)$. In Fig. 1 we show the experimental result of the tune measurement at the SPring-8 storage ring. In the experiment we varied the horizontal tune with the vertical one fixed so as to cross the difference resonance. Here the distance from resonance is estimated from the unperturbed tunes $\nu_{x, y}$, which are

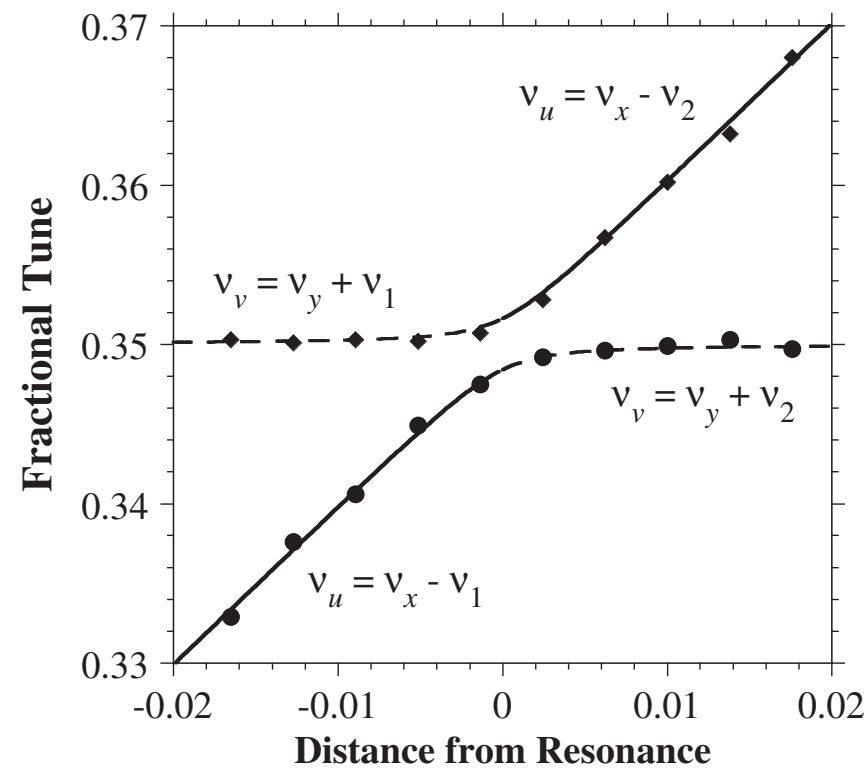

FIG. 1. Measured fractional tunes at the SPring-8 storage ring. The solid line indicated the expected horizontal tune, the dashed one corresponds to the vertical. 
calculated from the strengths of the quadrupole magnets without the error skew quadrupole field. Note that the minimum tune separation $\left|\nu_{u}-\nu_{v}\right|$ at $\Delta=0$ gives the strength of the coupling $|C|$. In the present case it is estimated to be 0.0032 .

\section{EQUILIBRIUM BEAM ENVELOPE}

Although the equilibrium beam emittances in the coupled motion have been derived by the Hamilton perturbation theory [3], we reproduce them in the present matrix formulation. In this section we denote the normal coordinates by $\mathbb{U}^{t}=\left(u, p_{u}, v, p_{v}\right)$, which is related to the original coordinate $\mathbb{X}$ by the symplectic rotation matrix $\boldsymbol{T}$ as

$$
\mathbb{U}=\boldsymbol{T} \mathbb{X} .
$$

The invariant amplitudes are straightforwardly defined in the normal coordinate since the oscillating motion completely decouples in the coordinate. Hence, the oscillating motion in the normal coordinate is simple betatron oscillation. As found from Eq. (25), we can regard the lattice functions of unperturbed motion as those of the eigenmodes of coupled motion. Following [6], we then define the invariant amplitudes

$$
a_{w}^{2}=\left(w, p_{w}\right)\left(\begin{array}{cc}
\gamma_{z} & \alpha_{z} \\
\alpha_{z} & \beta_{z}
\end{array}\right)\left(\begin{array}{c}
w \\
p_{w}
\end{array}\right) .
$$

Ignoring the vertical dispersion, we find that in the case of $\Delta>0$ the impact of the photon radiation on the normal modes is given by [6]

$$
\begin{aligned}
& \left(\begin{array}{c}
\delta u \\
\delta p_{u}
\end{array}\right)=-\frac{\epsilon}{E_{0}} \tau\left(\begin{array}{c}
\eta_{x} \\
\eta_{x}^{\prime}
\end{array}\right), \\
& \left(\begin{array}{c}
\delta v \\
\delta p_{v}
\end{array}\right)=-\frac{\epsilon}{E_{0}} \frac{\tau}{2 \nu_{1}} N_{y x}^{(c)}\left(\begin{array}{c}
\eta_{x} \\
\eta_{x}^{\prime}
\end{array}\right),
\end{aligned}
$$

where $\epsilon$ is the emitted photon energy, $E_{0}$ the nominal energy of the stored electron, and $\eta_{x}$ the horizontal dispersion function. The derivation of the beam envelope in the case of $\Delta<0$ is completely parallel to that of $\Delta>0$. If we put for $z$ and $p_{z}$ in Eq. (27) the above disturbance, we obtain the resulting amplitude $\delta a_{z}^{2}$ excited by the photon emission. Using the relation

$$
N_{y x}^{(c) t}\left(\begin{array}{cc}
\gamma_{y} & \alpha_{y} \\
\alpha_{y} & \beta_{y}
\end{array}\right) N_{y x}^{(c)}=|C|^{2}\left(\begin{array}{cc}
\gamma_{x} & \alpha_{x} \\
\alpha_{x} & \beta_{x}
\end{array}\right)
$$

we have

$$
\delta a_{u}^{2}=\tau^{2} \frac{\epsilon^{2}}{E_{0}^{2}} \mathcal{H}(s), \quad \delta a_{v}^{2}=\frac{\tau^{2}|C|^{2}}{4 \nu_{1}^{2}} \frac{\epsilon^{2}}{E_{0}^{2}} \mathcal{H}(s),
$$

where

$$
\mathcal{H}(s)=\left(\eta_{x}, \eta_{x}^{\prime}\right)\left(\begin{array}{cc}
\gamma_{x} & \alpha_{x} \\
\alpha_{x} & \beta_{x}
\end{array}\right)\left(\begin{array}{l}
\eta_{x} \\
\eta_{x}^{\prime}
\end{array}\right)
$$

Thus, we find the quantum excitation of the invariant amplitudes in the normal coordinate. Here, for the brevity, we neglect the difference between the damping partition numbers for the lateral directions, which is small in most cases. Then considering the stationary condition of the invariant amplitudes between the radiation damping and the excitation, we can simply derive the equilibrium emittances

$$
\begin{aligned}
& \varepsilon_{u} \equiv \frac{1}{2}\left\langle a_{u}^{2}\right\rangle=\tau^{2} \varepsilon_{0}=\frac{\nu_{1}}{\nu_{1}-\nu_{2}} \varepsilon_{0}, \\
& \varepsilon_{v} \equiv \frac{1}{2}\left\langle a_{v}^{2}\right\rangle=\frac{\tau^{2}|C|^{2}}{4 \nu_{1}^{2}} \varepsilon_{0}=-\frac{\nu_{2}}{\nu_{1}-\nu_{2}} \varepsilon_{0},
\end{aligned}
$$

where the symbol $\langle\cdot\rangle$ denotes the average over the photon fluctuation and the circumference, and $\varepsilon_{0}$ is the natural emittance [6]. Note that, as expected, we find

$$
\varepsilon_{u}+\varepsilon_{v}=\varepsilon_{0}
$$

which implies the share of the oscillating energy between the eigenmodes.

In electron storage rings, the equilibrium distributions are well described by the Gaussian. At the present case therefore the distribution function in the normal coordinate is given by

$$
\rho(\mathbb{U})=\frac{1}{4 \pi^{2} \varepsilon_{u} \varepsilon_{v}} \exp \left(-\frac{a_{u}^{2}}{2 \varepsilon_{u}}-\frac{a_{v}^{2}}{2 \varepsilon_{v}}\right) .
$$

The distribution function in the real space can be obtained by the pull-back from the normal coordinate $\mathbb{U}$ to the original one $\mathbb{X}$. Note that the Jacobian of the transformation is given by $\operatorname{Det}(\boldsymbol{T})$, and equal to 1 . After the pull-back, the projection of beam profile on the $x-y$ plane is derived by integrating the momenta $p_{x, y}$ out:

$$
\tilde{\rho}(x, y)=R \exp \left[-2 \pi \sqrt{\beta_{x} \beta_{y}} R F(x, y)\right],
$$

where $R$ is the normalization factor

$$
R=\frac{\left(\nu_{1}-\nu_{2}\right)^{2}}{2 \pi \varepsilon_{0} \sqrt{\beta_{x} \beta_{y}\left[2\left(\nu_{1}^{2}+\nu_{2}^{2}\right)-\frac{c_{r}^{2}}{|C|^{2}}\left(\nu_{1}+\nu_{2}\right)^{2}\right]}},
$$

and

$$
F(x, y)=\frac{1}{\beta_{x}} x^{2}-\frac{2 c_{r}\left(\nu_{1}+\nu_{2}\right)}{|C|^{2} \sqrt{\beta_{x} \beta_{y}}} x y+\frac{2\left(\nu_{1}^{2}+\nu_{2}^{2}\right)}{|C|^{2} \beta_{y}} y^{2} .
$$

The tilt angle of an ellipse $A x^{2}+B x y+C y^{2}=1$ is given by $\tan 2 \theta=B /(A-C)$. Hence, the tilt angle $\theta$ of the beam profile in the $x-y$ plane is

$$
\tan 2 \theta=\frac{2 c_{r} \sqrt{\beta_{x} \beta_{y}} \Delta}{2 \beta_{x} \Delta^{2}+\left(\beta_{x}-\beta_{y}\right)|C|^{2}} .
$$

The projected beam distribution on the horizontal or the vertical plane can be derived by integrating out the opposite coordinate, respectively. The integration of Eq. (29) 
over $y$ gives the horizontal beam distribution

$$
\rho_{x}(x)=\frac{1}{\sqrt{2 \pi} \sigma_{x}} \exp \left(-\frac{x^{2}}{2 \sigma_{x}^{2}}\right),
$$

where $\sigma_{x}$ is the horizontal beam size [3]

$$
\sigma_{x}^{2}=\frac{\Delta^{2}+\frac{1}{2}|C|^{2}}{\Delta^{2}+|C|^{2}} \beta_{x} \varepsilon_{0} .
$$

Similarly, integrating Eq. (29) with respect to the horizontal coordinate, we obtain the vertical beam size [3]

$$
\sigma_{y}^{2}=\frac{\frac{1}{2}|C|^{2}}{\Delta^{2}+|C|^{2}} \beta_{y} \varepsilon_{0}
$$

It is remarkable that the conservation of the sum of the equilibrium beam emittances

$$
\frac{\sigma_{x}^{2}}{\beta_{x}}+\frac{\sigma_{y}^{2}}{\beta_{y}}=\varepsilon_{0}
$$

is recovered [3].

Now we compare the formula for the beam profile with the experiment in the tune survey at the SPring- 8 storage ring. In the experiment, the beam profile is measured by the two-dimensional visible light interferometer [13] and by the x-ray beam profile monitor [14] with using synchrotron radiation from a bending magnet. The monitors are installed at the symmetric points with the same lattice func-

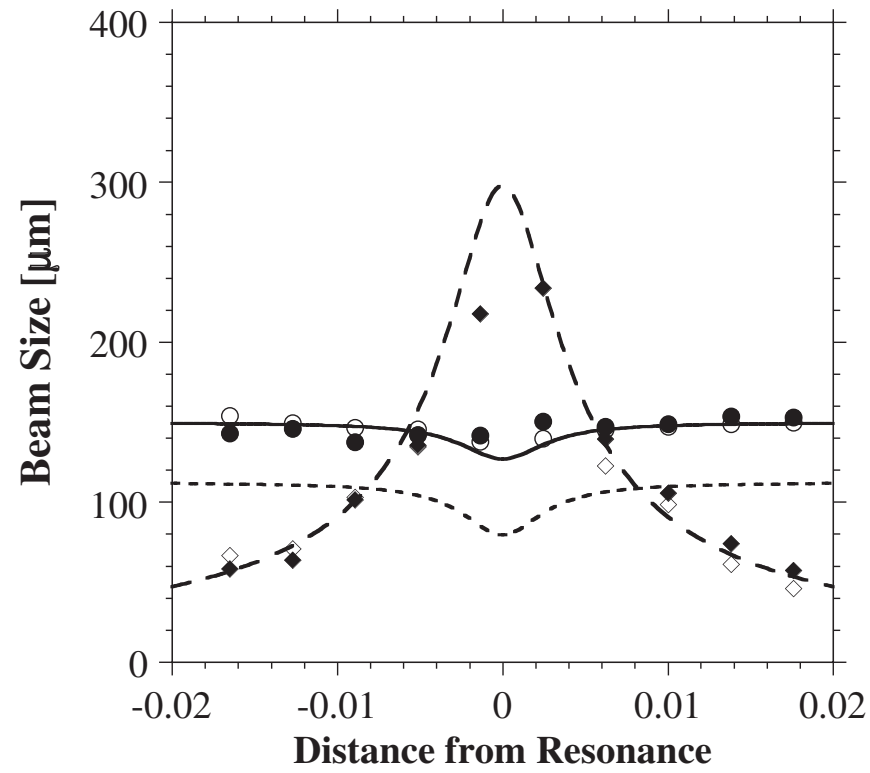

FIG. 2. Measured beam sizes at the SPring-8 storage ring. The solid and open circles denote the horizontal beam sizes measured by the x-ray beam profile monitor and by the visible light interferometer, respectively. The diamonds indicate the vertical ones. The solid (dotted) line represents the expected horizontal beam size calculated with (without) the contribution of the energy spread through the dispersion. The dashed line indicates the expected vertical beam size. tion. These monitors can observe the inclination of the beam profile in addition to the lateral beam spreads.

In Fig. 2 we plot the change of the beam sizes during the tune survey with crossing the difference resonance, where the circles (diamonds) indicate the measurement results of the horizontal (vertical) beam size. The expected horizontal (vertical) beam size from the present formula is plotted by the dotted (dashed) line. The horizontal and vertical betatron functions at the monitors are, respectively, 1.91 and $26.88 \mathrm{~m}$, the (calculated) natural emittance is $6.6 \mathrm{~nm} \mathrm{rad}$, the strength of the coupling driving term $|C|$ is estimated to be 0.0032 as mentioned in the previous section. The difference between the measurement and the calculation in the horizontal beam size comes from the contribution of momentum spread. Including the mean square radial spread due to the energy spread $\sigma_{\epsilon}$

$$
\sigma_{x \epsilon}=\eta_{x} \sigma_{\epsilon}
$$

we write the total horizontal beam size as

$$
\tilde{\sigma}_{x}^{2}=\sigma_{x}^{2}+\sigma_{x \epsilon}^{2}
$$

which is indicated by the solid line in Fig. 2. The dispersion function takes the value of $0.09 \mathrm{~m}$ at the source points of the monitors and the energy spread of the SPring- 8 storage ring is 0.0011 . The experimental data of the horizontal beam size measured by the beam profile monitor have no distinct dip represented by the theoretical line. This is because of the measurement error margin. Note that the

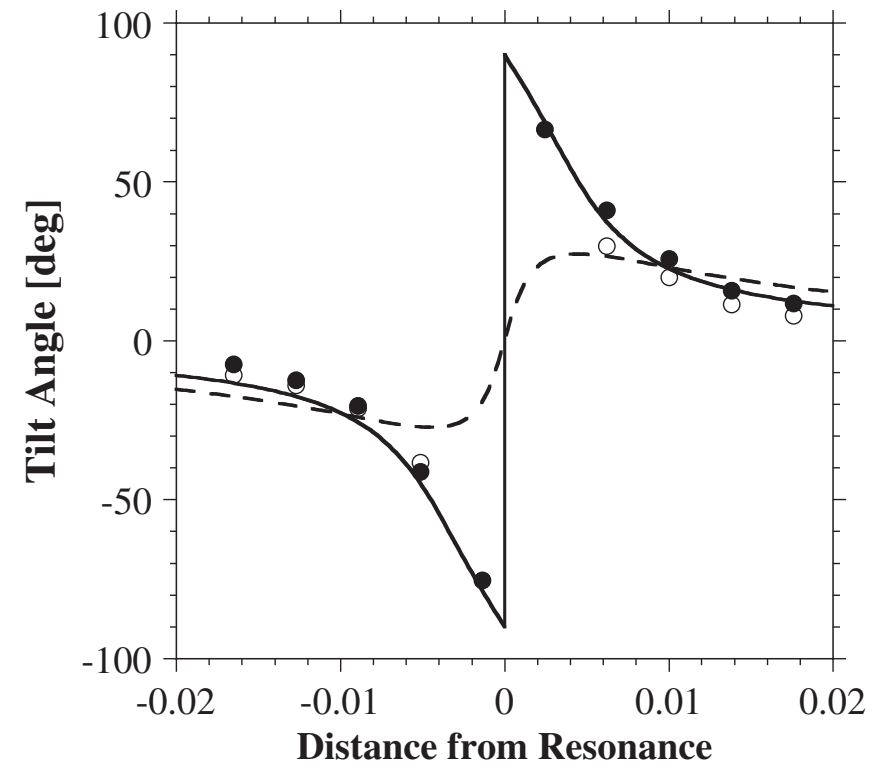

FIG. 3. Measured beam tilt at the SPring-8 storage ring. The full (open) circles denote the measurement result by the beam profile monitor (interferometer) and the solid line displays the expected inclination by the formula at the position of the monitors. The dotted line indicates the expected inclination at the center of a straight section. 
data measured by the interferometer has a clear dip like the theoretical expectation.

The measured tilt angle of the beam profile is shown in Fig. 3. The tilt angles expected by the formula (30) with the same lattice parameters to the above measurements are plotted by the solid line. The unknown real part of the coupling driving term $c_{r}$ is determined to be 0.002 by best fit, which lies in a physically consistent range $\left|c_{r}\right| \leq|C|$.

It is remarkable that the dependence of the tilt angle on the distance from the resonance shows significantly different behavior according to whether $\beta_{x}>\beta_{y}$ or $\beta_{x}<\beta_{y}$. For $\beta_{x}<\beta_{y}$ such as for the beam profile monitors of the SPring- 8 storage ring, the inclination of the beam ellipse reaches a right angle, $\pm 90^{\circ}$, on resonance as indicated by the solid line in Fig. 3. For $\beta_{x}>\beta_{y}$ the tilt angle never surpasses $\pm 45^{\circ}$ and becomes zero on resonance as shown by the dotted line. The betatron functions $\left(\beta_{x}, \beta_{y}\right)$ corresponding to the dotted line are $(24.47 \mathrm{~m}, 5.76 \mathrm{~m})$. The limitation comes from the fact that for the case of $\beta_{x}>\beta_{y}$ the denominator of the tilt angle representation (30) by no means crosses zero, i.e. $\tan 2 \theta$ never reaches the infinity, where the angle $\theta$ corresponds to $\pm 45^{\circ}$. It is another interesting property that the tilt angle changes the sign while crossing the resonance.

\section{SUMMARY}

In this paper we complete the matrix formulation of the Hamilton perturbation theory for the linearly coupled betatron motion. Hence, we give the analytical representation for the Teng parametrization for the transfer matrix of twodimensional coupled motion in the vicinity of a difference resonance. The normal modes of the coupled motion and the coupling matrix are explicitly represented by the coupling driving term and the unperturbed lattice functions.

The Teng parametrization includes the transformation matrix giving the normal coordinate, where the coupled betatron oscillation is decoupled. The beam emittances of each oscillation mode are then determined in the same way to the one-dimensional betatron motion. Thus, applying the formalism to an electron storage ring, we derive the analytical representation for the ellipse parameters of the equilibrium beam distribution in the real space.

\section{ACKNOWLEDGMENTS}

The author would like to thank Dr. H. Tanaka, Dr. K. Soutome, and Dr. J. Schimizu for helpful discussions. The author also would like to acknowledge Dr. S. Takano and Dr. M. Masaki for their experimental measurements of a beam profile.

[1] G. Guignard, CERN Report No. CERN 76-06, 1976.

[2] G. Guignard, CERN Report No. CERN ISR-BOM/77-43, 1977.

[3] G. Guignard, Phys. Rev. E 51, 6104 (1995).

[4] F. Willeke and G. Ripken, in Physics of Particle Accelerators, AIP Conf. Proc. No. 184 (America Institute of Physics, New York, 1989), p. 758.

[5] E. D. Courant and H. S. Snyder, Ann. Phys. (Leipzig) 3, 1 (1958).

[6] M. Sands, in Proceedings of International School of Physics Enrico Fermi, Course 46, Physics with Intersecting Storage Ring, edited by B. Touschek (Academic Press, San Diego, 1971), p. 257.

[7] L. C. Teng, FN-229, 1971.

[8] D. A. Edwards and L.C. Teng, in Proceedings of IEEE PAC, 1973, p. 855.

[9] D. Sagan and D. Robin, Phys. Rev. ST Accel. Beams 2, 074001 (1999).

[10] D. Sagan R. Meller, R. Littauer, and D. Robin, Phys. Rev. ST Accel. Beams 3, 092801 (2000).

[11] C.X. Wang and R. Calaga, in Proceedings of the European Particle Accelerator Conference, Lucerne, Switzerland, 2004 (EPAC 2004), p. 1470.

[12] R. Calaga, R. Tomás, and A. Franchi, Phys. Rev. ST Accel. Beams 8, 034001 (2005).

[13] M. Masaki and S. Takano, J. Synchrotron Radiat. 10, 295 (2003).

[14] S. Takano, M. Masaki, and H. Ohkuma, Nucl. Instrum. Methods Phys. Res., Sect. A 556, 357 (2006). 